\title{
REPRESENTAÇÕES SOCIAIS DA EDUCAÇÃO EM SAÚDE PELOS PROFISSIONAIS DA EQUIPE DE SAÚDE DA FAMÍLIA
}

\author{
SOCIAL REPRESENTATIONS OF HEALTH EDUCATION OF FAMILY HEALTH TEAM PROFESSIONALS
}

\author{
Maria Flávia Carvalho Gazzinelli ${ }^{1}$ \\ Rita de Cássia Marques ${ }^{2}$ \\ Denize Cristina de Oliveira ${ }^{3}$ \\ Maria Marta Amancio Amorim ${ }^{4}$ \\ Eduardo Gomes de Araújo ${ }^{5}$
}

Resumo Este estudo discute a forma como os profissionais da equipe de saúde da família pensam e representam a educação em saúde e a prática educativa que realizam. Os sujeitos da pesquisa $(\mathrm{n}=248)$ - médico, enfermeiro, auxiliar de enfermagem e agente comunitário de saúde de 20 unidades básicas de saúde de Belo Horizonte - foram entrevistados mediante a utilização da técnica de evocação livre e da teoria das representações sociais. Solicitou-se aos sujeitos que verbalizassem cinco palavras que lhes ocorressem imediatamente em relação à educação em saúde, classificando-as em positiva ou negativa. Utilizou-se o software Evoc e calculou-se a frequência simples de ocorrência, a média ponderada de ocorrência em função da ordem de evocação e a média das ordens ponderadas do conjunto dos termos evocados. Construiu-se o quadro de quatro casas composto do núcleo central, elementos de contraste e periféricos da representação. Médicos e enfermeiros compartilham conteúdos representacionais entre si, expressando representações sociais similares, ao apresentarem nos possíveis núcleos centrais 'capacitação' e 'prevenção'. Auxiliares de enfermagem e agentes comunitários de saúde se assemelham por meio dos possíveis elementos centrais 'educação' e 'prevenção'. O foco recai na transmissão de orientações com o objetivo de persuadir os indivíduos à adoção de modos de vida saudáveis. Palavras-chave educação em saúde; saúde da família; representações sociais.
Abstract This study discusses how the professionals on the family health team interpret and represent the health education and educational practice they perform. The subjects $(\mathrm{n}=248)$ - physicians, nurses, nurse aides, and community health workers from 20 basic health units in Belo Horizonte (southeastern Brazil) - were interviewed using the free recall technique and the theory of social representations. Subjects were asked to verbalize five words that came to their minds immediately when thinking about health education, rating them as positive or negative. The Evoc software was used and the simple frequency of occurrence, the weighted average of occurrence based on the order of recall, and the weighted average of all the terms mentioned were calculated. The four decimal frame comprising the central core and contrast and peripheral elements of representation was constructed. Physicians and nurses shared representational content among themselves, expressing similar social representations when presenting the possible central core of 'empowerment' and 'prevention.' Nursing aides and community health workers resemble each other through the possible central elements of 'education' and 'prevention.' The focus is on the conveyance of guidelines aimed at persuading individuals to adopt healthy lifestyles.

Keywords health education; family health; social representations. 


\section{Introdução}

As representações sociais são entendidas como uma forma de conhecimento elaborada e compartilhada socialmente, constituída de informações, de crenças, de opiniões e de atitudes a propósito de um dado objeto social, que contribui para a construção de uma realidade comum a um grupo social (Wagner, 2000). Elas são elaboradas pelo grupo social e reconstruídas pelo indivíduo em sua experiência interior. Apesar de serem compartilhadas, as representações sociais possuem caráter dinâmico, sendo negociadas por meio da interação social e da conversação, bem como modificadas ou adaptadas à medida que são incorporadas pelo indivíduo na sua concepção de mundo.

Assim, o estudo das representações sociais por meio da teoria das representações sociais permite analisar a interação entre o universo individual e o contexto social no qual os atores sociais estão inseridos (Oliveira, 1996). A teoria das representações sociais reconhece o indivíduo como um ser psicossocial, pois ele se apropria de um conhecimento, aplica nele o seu toque pessoal e o compartilha com o seu grupo de pertença, ou seja, o sujeito possui uma história pessoal com determinantes sociais e culturais (Moscovici, 2004).

Evidencia-se, portanto, uma estreita relação entre as representações sociais e o contexto de vida dos sujeitos. Os contextos histórico, ideológico e social, bem como a forma de pensar e agir dos grupos influenciam a construção, a partilha e a estabilidade das representações sociais (Marques et al., 2004). Reconhecer a educação em saúde, tema deste estudo, como objeto de representação social é entender como ela é elaborada pelos profissionais de saúde com base em suas crenças adotadas coletivamente com o objetivo de explicar sua experiência social.

A educação em saúde consiste no campo de teorias e práticas que se ocupa das relações entre o conhecimento e os processos de saúde e doença dos indivíduos e da coletividade. Essa construção de conhecimento é transversalizada por um possível diálogo entre o saber instituído, elaborado pela produção científica e sujeito a uma revisão permanente, e o senso comum, resultante da vivência cotidiana e baseado em relações perceptivas e afetivas, de significados próprios. Nesse processo, os sujeitos acabam produzindo, numa interface entre o individual e o coletivo, conhecimentos que são específicos e compartilhados, nos quais o fazer, a ação, tem papel preponderante.

Informações de vários estudos atestam a distância entre as práticas de educação em saúde no país, que permanecem com enfoque na pedagogia da transmissão da informação, cujas bases se situam na filosofia essencia- 
lista (Araújo, 2004; Gastaldo, 1997; Moura e Sousa, 2002; Wendhausen e Saupe, 2003) e as políticas públicas na saúde, que apresentam vários avanços conceituais. Outras pesquisas apontam como dificuldades das equipes de saúde da família na execução de práticas educativas a falta de infraestrutura - local adequado e tempo disponível -, de recursos didáticos e de formação pedagógica dos profissionais (Araújo, 2004; L'Abbate, 1994; Moura e Sousa, 2002; Wendhausen e Saupe, 2003). Importa destacar que, mesmo assumidos muitas vezes sem questionamento, esses conceitos e dificuldades vêm influenciando a concepção e a prática da educação em saúde na atualidade.

Este estudo, por conseguinte, está voltado para a compreensão do sistema de ideias, noções, crenças e atitudes daqueles que se envolvem com a educação em saúde, desafio que pode se tornar possível com a teoria das representações sociais (Abric, 2000; Sá, 2002). Portanto, pretende discutir a forma como os profissionais da equipe de saúde da família pensam e representam a educação em saúde e a prática educativa que realizam.

\section{Metodologia}

Este estudo 6 fundamenta-se no referencial da teoria do núcleo central ou abordagem estrutural das representações. Embora essa teoria privilegie procedimentos quantitativos, contém uma abordagem metodológica múltipla que associa aspectos qualitativos e quantitativos no levantamento e análise dos dados (Campos, 2003).

A teoria do núcleo central elaborada por Jean-Claude Abric em 1976, abordagem complementar da teoria das representações sociais, descreve a estruturação interna das representações estabilizadas, que se organiza em um sistema central e um sistema periférico, com características e funções distintas (Abric, 1994).

Os sujeitos da pesquisa foram selecionados com base em 68 equipes da Estratégia Saúde da Família (ESF), compostas de um médico, um enfermeiro, um auxiliar de enfermagem e um agente comunitário de saúde (ACS), de vinte unidades básicas de saúde (UBSs) do Distrito Sanitário Nordeste do município de Belo Horizonte. Os 248 profissionais da ESF eleitos assinaram o Termo de Consentimento Livre e Esclarecido, aprovado pelos Comitês de Ética em Pesquisa da Secretaria de Saúde do Município de Belo Horizonte e da Universidade Federal de Minas Gerais (UFMG). O perfil e frequência da clientela entrevistada estão descritos no quadro 1. 
Quadro 1

Perfil e frequência da clientela entrevistada: Distrito Sanitário Nordeste, Belo Horizonte/MG, 2006

\begin{tabular}{cccc}
\hline Variável & Categoria & $\begin{array}{c}\text { Sujeitos } \\
(\mathbf{n})\end{array}$ & $\begin{array}{c}\text { Frequência } \\
\text { (\%) }\end{array}$ \\
\hline Sexo & masculino & feminino & $16 \%$ \\
& & 208 & $84 \%$ \\
\hline Idade & $\leq$ a 40 anos & 106 & $43 \%$ \\
Tempo de & $>$ de 40 anos & 142 & $57 \%$ \\
PSF & $\leq$ a 3 anos & 96 & $40 \%$ \\
\hline Categoria & $>$ de 3 anos & 152 & $60 \%$ \\
\hline profissional & médico & 51 & $21 \%$ \\
& enfermeiro & 61 & $25 \%$ \\
& auxiliar de enfermagem & 68 & $27 \%$ \\
\hline
\end{tabular}

Fonte: Os autores.

A coleta de dados foi realizada sob a forma de entrevista individual, com duração média de 10 a 20 minutos, utilizando-se a técnica de evocação livre (Sá, 2002). O método da evocação ou associação livre permite colocar em evidência o universo semântico do objeto estudado, assim como a sua dimensão imagética, de forma mais rápida e dinâmica que outros métodos com igual objetivo, como a entrevista (Abric, 2001).

O entrevistador solicitou aos sujeitos que verbalizassem cinco palavras ou expressões que lhes ocorressem imediatamente em relação ao termo indutor 'educação em saúde', com posterior classificação e registro de cada palavra com os termos positivo $(+)$ ou negativo (-). Cada entrevistado enumerava, numa escala de 1 a 5, as evocações por ordem crescente de importância (Abric, 2003). Os dados foram posteriormente quantificados por evocação padronizada e, quando necessário, testados pelo teste de hipótese qui-quadrado.

O tratamento dos dados coletados foi realizado por meio do software Ensemble de Programmes Permettant l'Analyse des Évocations (Evoc), versão 2003 (Vergès, 2000). Para a construção do corpus de análise, cada palavra foi 
digitada em seu formato original, em duas planilhas Excel, uma na ordem em que as palavras foram lembradas (corpus evocado) e outra na ordem de importância atribuída pelos sujeitos (corpus hierarquizado), segundo modelo do software. Cada evocação do corpus original foi substituída por seu respectivo termo padrão, com base no dicionário. O corpus final foi formatado no padrão: número da entrevista; sexo; idade; tempo de ESF; profissão; 1 palavra 2 palavra 3 palavra 4 palavra 5 palavra. Com base em um dicionário de palavras produzidas pela população de sujeitos, o software calculou e informou, para cada termo indutor, a frequência simples de ocorrência de cada palavra, a média ponderada de ocorrência de cada palavra em função da ordem de evocação e a média das ordens ponderadas do conjunto dos termos evocados (Oliveira et al., 2005).

Com base nesses valores de corte, foram compostas as informações para a construção do chamado 'quadro de quatro casas', por meio do qual se discriminam o núcleo central, os elementos de contraste e os elementos periféricos da representação (Sá, 2002; Oliveira, 2005). Comparou-se a frequência e o valor médio da ordem de evocação de cada termo com os valores de corte dos quadrantes e procedeu-se à interpretação dos mesmos (Oliveira et al., 2005).

\section{Resultados e discussão}

\section{Estruturas representacionais da educação em saúde evocadas pelos profissionais da ESF}

O quadro 2 ilustra o quadro de quatro casas das evocações dos profissionais de saúde entrevistados que compõem a ESF - médico, enfermeiro, auxiliar de enfermagem e ACS. A ordem média das evocações (OME) foi igual a 3, proporcionando a divisão do quadro em 2 lados: direito, $\geq 3$, e esquerdo, $<3$. Ressalta-se que, quanto menor a média da ordem de evocações, mais importantes são os atributos elencados.

A frequência média de evocações (FME) foi igual a 36 e permitiu a divisão do quadro em mais 2 casas: superior, $\geq 36$, e inferior a $<36$. Destaca-se que quanto maior a frequência maior a importância do atributo. 
Quadro 2

Quadro das quatro casas do termo indutor 'educação em saúde' dos profissionais da Estratégia Saúde da Família: Distrito Sanitário Nordeste, Belo Horizonte/MG, 2006.

\begin{tabular}{|c|c|c|c|c|c|c|c|c|c|c|}
\hline \multirow{2}{*}{$\begin{array}{c}\mathrm{OME}^{1} \\
\mathrm{FME}^{2}\end{array}$} & \multicolumn{5}{|c|}{$<3,0$} & \multicolumn{5}{|c|}{$>3,0$} \\
\hline & $\begin{array}{c}\text { Termo } \\
\text { evocado }\end{array}$ & $\mathrm{FE}^{3}$ & $\mathrm{OME}^{1}$ & Atitude & $\mathrm{p}$ & $\begin{array}{c}\text { Termo } \\
\text { evocado }\end{array}$ & $\mathrm{FE}^{3}$ & $\mathrm{OME}^{1}$ & Atitude & $p$ \\
\hline \multirow{4}{*}{$>36$} & Prevenção & 77 & 2,208 & + & $<0,01$ & & & & & \\
\hline & Educação & 48 & 2,667 & + & $<0,01$ & & & & & \\
\hline & Orientação & 44 & 2,000 & + & $<0,01$ & & & & & \\
\hline & Capacitação & 40 & 2,850 & + & $<0,01$ & & & & & \\
\hline \multirow{6}{*}{$<36$} & Conhecimento & 35 & 2,600 & + & $<0,01$ & Condições-vida & 32 & 3,563 & + & $<0,01$ \\
\hline & Informação & 32 & 1,875 & + & $<0,01$ & Saúde & 29 & 3,448 & + & $<0,01$ \\
\hline & Grupos & 32 & 2,063 & + & $<0,01$ & Troca & 23 & 3,870 & + & $<0,01$ \\
\hline & Instrução & 30 & 2,233 & $+/-$ & 0,715 & & & & & \\
\hline & Não funciona & 23 & 2,043 & - & $<0,01$ & & & & & \\
\hline & Importante & 22 & 2,545 & + & $<0,01$ & & & & & \\
\hline
\end{tabular}

Fonte: Os autores.

Siglas: $10 M E$ (ordem média de evocações); ${ }^{2} \mathrm{FME}$ (frequência média de evocações); ${ }^{3} \mathrm{FE}$ (frequência de evocação).

No quadrante superior esquerdo, que agrupa os elementos mais frequentes e mais importantes (Oliveira et al., 2005), observa-se a presença do atributo conceitual 'prevenção', a maior frequência de todas as evocações, e 'orientação' e 'prevenção', que apresentam, respectivamente, o primeiro e o segundo lugares em importância em função da ordem de evocação, seguidos pelo atributo conceitual 'educação' e pelo atributo profissional 'capacitação'. Esse quadrante representa o núcleo central que é determinado pela natureza do objeto representado, pelas relações que o grupo mantém com o objeto e pelo sistema de valores e normas que norteiam o grupo. O núcleo central possui duas funções: geradora - cria ou transforma elementos da representação - e organizadora - unifica e estabiliza as representações. Possui uma dimensão quantitativa e outra qualitativa - dando significado à representação -, e também tem um caráter normativo - expressa o normal, mas não o que é correto.

No quadrante superior direito, onde se encontram os elementos periféricos mais importantes - primeira periferia (Oliveira et al., 2005) -, destaca-se a ausência de atributos, o que parece indicar uma representação com um conteúdo mais consolidado em seu núcleo central. Os elementos periféricos possuem as funções de concretização - ancoragem e regulação -, mobilidade, evolução e defesa da representação (Abric, 2000). São esquemas de 
funcionamento da representação, pois podem prescrever comportamentos, além de permitirem a modulação das representações e das condutas, protegendo, assim, o núcleo central. Os elementos periféricos também são ditos condicionais, uma vez que expressam o não frequente, o excepcional, mas não o anormal.

Observam-se no quadrante inferior esquerdo elementos que apresentam baixa frequência, mas são considerados importantes pelos sujeitos; pela ordem de evocação, a presença do atributo profissional 'conhecimento' e do conceitual 'informação', com maior frequência e menor ordem de evocação, respectivamente. O conteúdo conceitual é reforçado pelo elemento 'grupos'. Observam-se também os atributos avaliativos 'não funciona' e 'importante', além do atributo social 'instrução'. A zona de contraste revela elementos que reforçam as noções presentes no núcleo central ou na primeira periferia, ou, ainda, a existência de um subgrupo minoritário portador de uma representação diferente (Oliveira et al., 2005). Observam-se os elementos 'informação' e 'instrução' reforçando o modelo pedagógico diretivo no núcleo central, representado pelos atributos 'orientação' e 'educação'. Já o atributo 'grupos' aponta para uma educação em saúde afinada com as diretrizes da ESF, podendo configurar-se como subgrupo, pois tem um sentido diferente do restante da representação. Resultados similares foram encontrados por Pinafo et al. (2011), ao concluírem que as percepções de educação em saúde dos profissionais de saúde de uma ESF encontraram-se fortemente embasadas no modelo de transmissão de informação.

Dentre os elementos que se situam no quadrante inferior direito, e constituem os menos frequentes e menos importantes de toda a representação (Oliveira et al., 2005), encontramos os atributos sociais 'condições-vida' e 'saúde', além do atributo conceitual 'troca'. Essas palavras não acrescentam por si só sentidos novos à representação, reforçando as dimensões presentes entre o elemento de contraste, 'grupos'.

Em relação à análise de atitudes, observa-se um predomínio de atitudes positivas em todos os quadrantes. Atitudes negativas foram observadas somente entre os elementos de contraste em relação ao atributo 'não funciona'. Destaca-se no atributo 'instrução' a ausência de associação estatística $(p=0,72)$ entre os julgamentos expressos pelos sujeitos, não havendo diferença entre as frequências atribuídas ao positivo e ao negativo. Em todas as outras evocações, as associações foram estatisticamente significativas $(\mathrm{p}<0,01)$.

\section{Estruturas representacionais da educação em saúde evocadas pelos médicos}

Em relação ao corpus dos médicos, a média das ordens de evocação foi 3 e a frequência média de evocação igual a 13 (quadro 3). 
Dentre as palavras presentes no quadrante superior esquerdo, destacam-se 'capacitação' e 'prevenção', com maior frequência e menor ordem de evocação, respectivamente, como prováveis elementos centrais dessa representação. Observa-se que o possível núcleo central da representação social dos médicos está próximo do conjunto de profissionais (quadro 2), possuindo, nesse sentido, elementos semelhantes, como 'capacitação', reforçado por 'informação', 'educação' e 'prevenção'. O modelo hegemônico de educação em saúde mantém-se, com o enfoque na prevenção de doenças, na valorização do conhecimento científico dos profissionais em saúde e na prescrição de hábitos e condutas por meio da transmissão de informações, o que já foi encontrado em outros estudos com médicos (Alvarenga, 2005; Alves e Nunes, 2006).

Observa-se a ausência de atributos no quadrante superior direito, onde estariam os prováveis elementos periféricos. Já no quadrante inferior direito, destaca-se como segunda periferia a palavra 'equipe'.

Nos possíveis elementos de contraste, destacam-se as palavras 'grupos' e 'informação', respectivamente com maior frequência e menor ordem de evocação. Em seguida, foram evocados os atributos 'instrução', 'educação' e 'não funciona'. Possivelmente esses atributos evocados apontem para um menor comprometimento dos médicos com os princípios norteadores da ESF, principalmente no que se refere a estratégias educativas mais interacionistas e ao trabalho interdisciplinar. Alguns estudos corroboram esses achados quando afirmam que as atividades de educação continuada para os médicos na atenção básica ainda se concentram na abordagem diagnóstica e terapêutica, com poucas oportunidades de reflexão crítica das diretrizes do programa, que muitas vezes são apresentadas apenas por meio de documentos oficiais e formais (Alvarenga, 2005).

Quadro 3

Quadro das quatro casas do termo indutor 'educação em saúde' dos médicos da Estratégia Saúde da Família: Distrito Sanitário Nordeste, Belo Horizonte/MG, 2006.

\begin{tabular}{ccccccc}
\hline $\mathrm{OME}^{1}$ & \multicolumn{3}{c}{$<3,0$} & \multicolumn{3}{c}{$>3,0$} \\
\hline $\mathrm{FME}^{2}$ & Termo evocado & $\mathrm{FE}^{3}$ & $\mathrm{OME}^{1}$ & Termo evocado & $\mathrm{FE}^{3}$ & $\mathrm{OME}^{1}$ \\
\hline \multirow{2}{*}{$>13$} & Capacitação & 31 & 2,774 & & & \\
& Prevenção & 13 & 1,846 & & & \\
\hline \multirow{3}{*}{$<13$} & Grupos & 11 & 2,182 & Equipe & 11 & \multirow{2}{*}{ (1) } \\
& Informação & 10 & 1,700 & & & \\
& Instrução & 10 & 2,400 & & & \\
& Educação & 9 & 2,778 & & & \\
& Não funciona & 8 & 2,125 & & & \\
\end{tabular}

Fonte: Os autores.

Siglas: $10 M E$ (ordem média de evocações); ${ }^{2} \mathrm{FME}$ (frequência média de evocações); ${ }^{3} \mathrm{FE}$ (frequência de evocação). 
Estruturas representacionais da educação em saúde evocadas pelos enfermeiros

A frequência média de evocação dos enfermeiros foi igual a 12 (quadro 4). Dentre as palavras presentes no quadrante superior esquerdo, destacam-se 'capacitação' e 'prevenção', respectivamente com maior frequência e menor ordem de evocação, como os atributos mais importantes dentre os prováveis elementos centrais dessa representação. Esses atributos são seguidos pela palavra 'educação'.

Observa-se no quadrante superior direito a incorporação da palavra 'troca' como possível elemento periférico. Já no quadrante inferior direito, destacam-se como prováveis constituintes de uma segunda periferia as palavras 'experiência' e 'discussão', respectivamente com maior frequência e menor ordem de evocação, seguidas por 'condições-vida', 'vínculo' e 'participação'. Nos possíveis elementos de contraste, presentes no quadrante inferior esquerdo, destaca-se a palavra 'orientação'.

Observa-se uma semelhança no enfoque do modelo pedagógico diretivo ('educação', com reforço de 'orientação') voltado para a 'prevenção' encontrada entre os possíveis elementos centrais evocados pelos enfermeiros com as representações até aqui estudadas. Essa abordagem diretiva e preventivista da educação em saúde decorre, em grande parte, do enfoque clínico individual ainda presente na formação dos enfermeiros, enfoque que privilegia aspectos biológicos em detrimento dos sociais, emocionais e comportamentais (Silva et al., 2006).

O processo de ensino-aprendizagem deverá ter um olhar mais atento para a abordagem diretiva e preventivista, a fim de que os programas de educação e saúde não se limitem a iniciativas que visem apenas informar a população (Amancio, Carvalho e Rodrigues, 2012). Os valores, os costumes e os modelos sociais que conduzam a práticas educativas libertadoras devem ser considerados (Pinafo et al., 2011).

\section{Quadro 4}

Quadro das quatro casas do termo indutor 'educação em saúde' dos enfermeiros da Estratégia Saúde da Família: Distrito Sanitário Nordeste, Belo Horizonte/MG, 2006.

\begin{tabular}{|c|c|c|c|c|c|c|}
\hline \multirow{2}{*}{$\frac{\mathrm{OME}^{1}}{\mathrm{FME}^{2}}$} & \multicolumn{3}{|c|}{$<3,0$} & \multicolumn{3}{|c|}{$>3,0$} \\
\hline & Termo evocado & $\mathrm{FE}^{3}$ & $\mathrm{OME}^{1}$ & Termo evocado & $\mathrm{FE}^{3}$ & $\mathrm{OME}^{1}$ \\
\hline \multirow{3}{*}{$>12$} & Capacitação & 23 & 2,391 & Troca & 17 & 3,882 \\
\hline & Prevenção & 14 & 2,357 & & & \\
\hline & Educação & 12 & 2,583 & & & \\
\hline \multirow{5}{*}{$<12$} & Orientação & 10 & 1,600 & Experiência & 11 & 3,818 \\
\hline & & & & Condições-vida & 10 & 3,714 \\
\hline & & & & Vínculo & 9 & 3,444 \\
\hline & & & & Discussão & 7 & 3,200 \\
\hline & & & & Participação & 7 & 3,571 \\
\hline
\end{tabular}

Fonte: Os autores.

Siglas: ${ }^{O O M E}$ (ordem média de evocações); ${ }^{2} \mathrm{FME}$ (frequência média de evocações); ${ }^{3} \mathrm{FE}$ (frequência de evocação). 
Ressalta-se, no entanto, a incorporação de elementos referentes à perspectiva interacionista da educação em saúde na primeira periferia, com 'troca', reforçado por 'experiência' e 'discussão' na segunda periferia. Diversos estudos concordam que a abordagem interacionista, com destaque para as atividades em grupo, é a mais apropriada para as práticas educativas do enfermeiro na atenção básica (Alves, 2005; Silva et al., 2006).

\section{Estruturas representacionais da educação em saúde evocadas pelos auxiliares de enfermagem}

Em relação ao corpus formado pelas evocações dos auxiliares de enfermagem, a frequência média de evocação foi igual a 13 (quadro 5). Dentre as palavras presentes no quadrante superior esquerdo, destacam-se 'prevenção' e 'orientação', respectivamente com maior frequência e menor ordem de evocação, seguidas por 'educação'.

\begin{tabular}{|c|c|c|c|c|c|c|}
\hline \multicolumn{7}{|c|}{$\begin{array}{l}\text { Quadro das quatro casas das evocações do termo indutor 'educação em saúde' dos auxiliares de } \\
\text { enfermagem da Estratégia Saúde da Família: Distrito Sanitário Nordeste, Belo Horizonte/MG, } 2006 .\end{array}$} \\
\hline $\mathrm{OME}^{1}$ & \multicolumn{3}{|c|}{$<3,0$} & \multicolumn{3}{|c|}{$>3,0$} \\
\hline $\mathrm{FME}^{2}$ & Termo evocado & $\mathrm{FE}^{3}$ & $\mathrm{OME}^{1}$ & Termo evocado & $\mathrm{FE}^{3}$ & $\mathrm{OME}^{1}$ \\
\hline \multirow{3}{*}{$>13$} & Prevenção & 27 & 2,259 & & & \\
\hline & Orientação & 22 & 2,227 & & & \\
\hline & Educação & 13 & 2,462 & & & \\
\hline \multirow{4}{*}{$<13$} & Informação & 10 & 1,700 & Condições-vida & 12 & 3,583 \\
\hline & Difícil & 10 & 2,500 & Ensinar & 9 & 3,000 \\
\hline & Instrução & 8 & 2,250 & Recursos & 9 & 3,556 \\
\hline & Importante & 8 & 2,500 & & & \\
\hline
\end{tabular}

Fonte: Os autores.

Siglas: 1OME (ordem média de evocações); 2FME (frequência média de evocações); 3FE (frequência de evocação).

Observa-se a ausência de atributos no quadrante superior direito, onde se situariam os prováveis elementos periféricos. Já no quadrante inferior direito, destacam-se como prováveis constituintes de uma segunda periferia as palavras 'condições-vida' e 'ensinar', respectivamente com maior frequência e menor ordem de evocação, seguidas por 'recursos'. 
Nos possíveis elementos de contraste, presentes no quadrante inferior esquerdo, destaca-se a palavra 'informação', com maior frequência e menor ordem de evocação, seguida por 'difícil', 'instrução' e 'importante'.

Ressalta-se a incorporação de um conteúdo negativo na avaliação da prática do educador, no entendimento de que, apesar de reconhecerem a 'importância' da educação em saúde, para os auxiliares de enfermagem é uma prática 'difícil', principalmente pela falta de 'recursos'. O foco nos recursos materiais e como dificultador das atividades do auxiliar de enfermagem na ESF também foi identificado em outros estudos (Marsiglia, 2006; Shimizu et al., 2004). Essa dificuldade em realizar práticas educativas também pode ser explicada pelo fato dessa categoria profissional estar mais habituada a procedimentos técnicos em serviços hospitalares, com menor experiência e capacitação específica na atenção básica (Marsiglia, 2006).

\section{Estruturas representacionais da educação em saúde evocadas pelos agentes comunitários de saúde}

Em relação ao corpus formado pelas evocações dos agentes comunitários de saúde com base no termo indutor 'educação em saúde' a frequência média de evocação foi igual a 12 (quadro 6).

Dentre as palavras presentes no quadrante superior esquerdo, destacam-se 'prevenção' e 'grupos', respectivamente com maior frequência e menor ordem de evocação. Seguem-se as palavras 'educação' e 'instrução'. Para efeito de análise, não podemos afirmar que essa evocação pertença ao possível núcleo central, tendo em vista o desvio-padrão, aqui representado por sua frequência (12) ser igual à frequência média (12) da análise.

A presença do atributo 'instrução' associado a 'educação' entre os possíveis elementos centrais, reforçando a centralidade do modelo diretivo da educação, aparece nessa representação. O contato constante com a comunidade, da qual também fazem parte, onde as atividades educativas estão sempre presentes, pode explicar a incorporação da baixa instrução da população como elemento central.

Observa-se no quadrante superior direito a incorporação, como possível elemento periférico, da palavra 'saúde'. Já no quadrante inferior direito, destacam-se como prováveis constituintes de uma segunda periferia as palavras 'alimentação' e 'comunidade', respectivamente com maior frequência e menor ordem de evocação. 
Quadro 6

\begin{tabular}{|c|c|c|c|c|c|c|}
\hline \multirow{2}{*}{$\begin{array}{c}\begin{array}{l}\text { Quadro } \\
\text { da Estrat }\end{array} \\
\mathrm{OME}^{1} \\
\mathrm{FME}^{2}\end{array}$} & \multicolumn{3}{|c|}{$<3,0$} & \multicolumn{3}{|c|}{$>3,0$} \\
\hline & Termo evocado & $\mathrm{FE}^{3}$ & $\mathrm{OME}^{1}$ & Termo evocado & $\mathrm{FE}^{3}$ & $\mathrm{OME}^{1}$ \\
\hline \multirow{4}{*}{$>12$} & Prevençăo & 23 & 2,261 & Saúde & 18 & 3,333 \\
\hline & Educação & 14 & 2,857 & & & \\
\hline & Grupos 3 & 12 & 2,000 & & & \\
\hline & Instrução & 12 & 2,083 & & & \\
\hline \multirow{4}{*}{$<12$} & Importante & 9 & 2,333 & Alimentação & 11 & 4,182 \\
\hline & Informação & 9 & 2,333 & Comunidade & 9 & 3,000 \\
\hline & Não funciona & 8 & 1,750 & & & \\
\hline & Atenção & 8 & 2,875 & & & \\
\hline
\end{tabular}

Fonte: Os autores.

Siglas: 1OME (ordem média de evocações); 2 FME (frequência média de evocações); ${ }^{3} \mathrm{FE}$ (frequência de evocação).

Nos possíveis elementos de contraste, presentes no quadrante inferior esquerdo, observam-se as palavras 'importante', 'informação' e 'não funciona', as duas primeiras com maior frequência e a última com menor ordem de evocação, seguidas por 'atenção'.

Ressalta-se a incorporação do paradigma da promoção da saúde na primeira periferia, por meio do atributo 'saúde', reforçado pelas palavras 'comunidade' e 'alimentação'. Nesse estudo, o ACS foi a única categoria profissional que evocou elementos cujo enfoque recai sobre essa dimensão. Esse achado contrasta com outros estudos, que destacam como principal atribuição do ACS a visita domiciliar para a vigilância de riscos e o controle da aderência aos cuidados de saúde propostos pelo médico e pelo enfermeiro, mas ressaltam sua pouca identificação com as ações de promoção da saúde, incluídas aí as atividades educativas (Pedrosa e Teles, 2001; Nunes et al., 2002; Silva e Dalmaso, 2002).

\section{Considerações finais}

As afinidades conceituais dos grupos de médicos e enfermeiros parecem indicar que essas categorias profissionais pertencem a grupos sociais semelhantes, que compartilham conteúdos representacionais entre si, expressando representações sociais similares, pois apresentam em seus possíveis núcleos centrais os atributos 'capacitação' e 'prevenção'. Essa convergência conceitual pode estar associada ao fato de a formação desses profissionais ainda estar voltada 
para a valorização do saber científico, sob a influência do modelo biomédico, e também pelo fato de a representação social da educação em saúde se organizar em torno de um possível núcleo central, cujo enfoque recai na perspectiva do modelo pedagógico diretivo, voltado para a prevenção de doenças.

Já auxiliares de enfermagem e agentes comunitários de saúde se assemelham por meio dos possíveis elementos centrais 'educação' e 'prevenção'. Nessa perspectiva, também relacionada ao modelo diretivo e preventivo de educação em saúde, o foco recai na transmissão de orientações, com o objetivo de persuadir os indivíduos para a adoção de modos de vida saudáveis, a fim de evitarem o adoecimento.

A ideia presente nos possíveis elementos centrais citados pelas classes de profissionais - 'prevenção', 'orientação' e 'educação' - ancora-se nos pressupostos do sanitarismo-campanhista, modelo assistencial predominante nas primeiras décadas do século XX, conhecido pelo caráter intervencionista das práticas educativas em saúde. Sob sua influência, conforme observado no presente estudo, a educação em saúde continua objetivando a prevenção da doença por meio da transmissão de informações relativas à higiene e aos hábitos saudáveis. Essa similaridade no conteúdo dos campos representacionais das diferentes categorias profissionais ocorre provavelmente porque, na educação em saúde, as representações se apresentam como campo socialmente estruturado.

Outra dimensão bastante presente entre os possíveis elementos centrais diz respeito à valorização do conhecimento científico do profissional que atua na educação em saúde, em detrimento do saber popular da população, que é desvalorizado ou mesmo ignorado. Essa dimensão foi evocada por meio das palavras 'capacitação' e 'instrução', e parece ancorar-se no modelo médico-assistencial privatista, que permanece hegemônico desde meados dos anos 1960 e que prioriza uma assistência em saúde especializada, individual e curativa, focada na abordagem biomédica.

A educação em saúde representada sob esse aspecto permanece como uma prática social que deve ser realizada por profissionais que detêm o conhecimento científico, pressupondo a aceitação social da ciência médica como fonte legítima da verdade e definidora dos modos de vida e comportamentos que podem ser considerados corretos para que os indivíduos se tornem saudáveis.

Esses achados reforçam as características atribuídas por Abric (2000) ao núcleo central, formado por elementos ligados à história e à memória do grupo social, os quais apresentam resistência às mudanças e conferem, às representações, identidade e estabilidade. Além disso, constatamos também, na maioria dos profissionais da ESF, a presença de elementos no sistema periférico que atuam na defesa do sistema central, protegendo-o de possíveis transformações que provocariam uma alteração completa na representação. 
Uma das possíveis rupturas encontradas foi observada somente no campo representacional dos enfermeiros. Esses profissionais da saúde incorporaram no seu sistema periférico elementos relacionados com o modelo pedagógico interacionista, por meio dos elementos 'experiência' e 'discussão'. A educação em saúde representada nessa perspectiva parece se ancorar na educação popular, que começa a se estruturar como corpo teórico e prática social no final da década de 1950 com Paulo Freire. Seus pressupostos, assimilados nas ações de saúde, após sucessivas reformas nas políticas de saúde durante a década de 1970, propõem uma nova articulação entre educação e saúde, considerando as condições de vida da população, em contraponto à ênfase na causalidade biológica do processo saúde-doença.

Outra ruptura se fez presente por meio da perspectiva promoção da saúde somente no campo representacional dos agentes comunitários em saúde. Essa dimensão foi incorporada na primeira periferia, mediante palavras 'saúde' e 'alimentação' e 'promoção'.

A educação em saúde com esse enfoque se ancora nos ideais da vigilância da saúde, modelo assistencial que se desenvolveu a partir da reforma sanitária e da introdução da saúde como direito na Constituição de 1988. Essa abordagem objetiva que as pessoas exercitem poder sobre sua própria saúde. De acordo com esse referencial, não é mais possível definir limites precisos entre saúde e doença, pois essas se referem ao modo como as pessoas e as populações vivem, portanto relaciona-se com os contextos histórico, econômico e social, em um dado tempo e um determinado espaço geográfico. E a educação em saúde constitui uma prática que busca capacitar indivíduos e grupos a se auto-organizarem para desenvolver ações de promoção à saúde baseadas nas suas próprias prioridades.

Esses achados podem ser relacionados com outra função do sistema periférico, na abordagem estrutural das representações sociais. Segundo Abric (2000), esses elementos se localizam ao redor do núcleo central e realizam a conexão do sistema central com a realidade cotidiana. São eles que dão mobilidade e flexibilidade às representações sociais, regulando e adaptando o núcleo central às necessidades dos indivíduos e grupos sociais.

É preciso reconhecer que o processo de mudança de uma representação social é lento. Primeiramente, são transformados os elementos periféricos da representação, para somente depois os elementos centrais serem modificados, o que pode não ocorrer. Isso significa que as representações se transformam mais lentamente do que as oscilações das práticas, ou mesmo das realidades sociais, políticas e econômicas (Oliveira et al., 2005).

A utilização da abordagem estrutural das representações, elaborada por Jean-Claude Abric, e da técnica do quadro de quatro casas de Vergès, integradas à tecnologia da informática, mostraram-se importante instrumento facilitador da análise da estrutura e da organização de uma representação 
social. A técnica da associação livre, além de possibilitar o alcance dos elementos que constituem o universo semântico do termo ou do objeto estudado, devido ao caráter espontâneo, permitiu a organização dos dados dentro do software para uma análise mais criteriosa.

Solicitar aos próprios sujeitos que classificassem as palavras evocadas com os termos positivo ou negativo facilitou o acesso mais direto às suas atitudes em relação ao objeto estudado. Essa estratégia permitiu detectar, inclusive, variações importantes que se perderiam se essa classificação ficasse a cargo do pesquisador. Isso ocorreu em relação à palavra instrução, a única que não apresentou diferença estatística na atribuição de positivo e negativo, o que possibilitou o acesso ao seu duplo significado, ora com valor negativo, constatando a baixa instrução dos indivíduos, ora com valor positivo, como sinônimo de educação.

Uma limitação observada na análise dos dados se refere ao caráter extremamente objetivo do conteúdo semântico levantado por meio das evocações livres. Mesmo com a utilização do dicionário de padronização, a interpretação das palavras evocadas pelos sujeitos foi dificultada por não conseguirmos diferenciar possíveis conteúdos polissêmicos. Isso ocorreu em relação à palavra 'conhecimento', que tanto poderia referir-se à expressão 'ter conhecimento para' quanto poderia contrapor-se ao atributo 'informação', o que indicaria uma oposição epistemológica de grande valia para a análise.

O conteúdo das representações sociais analisadas neste estudo aponta para a necessidade de repensar os modelos pedagógico e assistencial que referendam as práticas educativas em saúde da ESF a fim de que a promoção da saúde possa efetivamente compor o rol de elementos considerados na definição das ações dos serviços e dos profissionais. Para tanto, toda prática educativa pode se beneficiar do fato de estar respaldada num projeto construído coletivamente pelos sujeitos envolvidos que explicite a sua concepção de sociedade, educação e trabalho, e apresente de forma clara não apenas o que se deseja alcançar com o processo educativo, mas também os meios para a concretização de tais objetivos.

Além disso, para a efetivação do novo modelo de saúde é indispensável que projetos de capacitação para as equipes da ESF facilitem a construção de sujeitos autônomos e críticos. Para que esse processo aconteça, devem levar-se em conta as representações sociais dos profissionais envolvidos, para entender como esses sujeitos reelaboram as diretrizes para as práticas educativas na ESF, a fim de que se estabeleçam algumas das condições favoráveis à superação do caráter meramente instrumental da capacitação cujos princípios se apoiam no saber científico.

É preciso também elevar o entendimento de formação profissional a uma experiência que propicie ao profissional o contato com modelos pedagógicos mais interacionistas - experiência entendida aqui como campo 
em que se entrecruzam representações e práticas, subjetividade e objetividade, pensamento e ação, corpo e mente (Gazzinelli e Penna, 2006). Somente assim os processos de capacitação tornam-se uma construção compartilhada de um conhecimento que parte da experiência e das práticas dos sujeitos envolvidos, buscando intervenções nas relações sociais que vão influenciar a qualidade de práticas educativas e, consequentemente, produzir outras representações.

Sabe-se que investir em ações que visem à transformação das representações sociais da educação em saúde, em qualquer contexto, é um processo longo, como plantar uma árvore.

\section{Colaboradores}

Maria Flávia Carvalho Gazzinelli, Rita de Cássia Marques, Denize Cristina de Oliveira, Maria Marta Amancio Amorim participaram da revisão crítica do texto; Eduardo Gomes de Araújo participou da coleta, análise dos dados e elaboração do artigo.

\section{Notas}

1 Universidade Federal de Minas Gerais, Belo Horizonte, Minas Gerais, Brasil.

Doutora em Educação pela Universidade Federal da Bahia.

$<$ flaviagazzinelli@enf.ufmg.br>

Correspondência: Rua Alfredo Balena, 190, Santa Efigênia, CEP 96020-220, Belo Horizonte, Minas Gerais, Brasil.

2 Universidade Federal de Minas Gerais, Belo Horizonte, Minas Gerais, Brasil.

Doutora em História pela Universidade Federal Fluminense. <rmarques@enf.ufmg.br>

3 Universidade Estadual do Rio de Janeiro, Rio de Janeiro, RJ, Brasil.

Doutora em Saúde Pública pela Universidade de São Paulo e pós-doutora em Psicologia Social pela École des Hautes Études em Sciences Sociales, Paris, França.

$<$ denize.oliveira@pq.cnpq.br>

4 Centro Universitário UNA, Belo Horizonte, Minas Gerais, Brasil.

Doutora em Enfermagem pela Universidade Federal de Minas Gerais.

$<$ martamorim@hotmail.com> 
5 Mestre em Enfermagem pela Universidade Federal de Minas Gerais, Belo Horizonte, Minas Gerais, Brasil. <eduardogaraujo@gmail.com>

6 Este artigo é parte da dissertação de mestrado do Curso de Pós-graduação em Enfermagem da Escola de Enfermagem da Universidade Federal de Minas Gerais, intitulada Educação em saúde: abordagem estrutural das representações sociais de profissionais do Programa de Saúde da Família, de autoria de Eduardo Gomes de Araújo.

\section{Referências}

ABRIC, Jean-Claude. Les representations sociales: aspects théoriques. In:

(Org.). Pratiques sociales et représentations. Paris: Presses Universitaires de France. 1994. p. 11-37.

A abordagem estrutural das representações sociais. In: MOREIRA, Antônia Silva Paredes; OLIVEIRA, Denise C. (Org.). Estudos interdisciplinares de representação social. 2 ed. Goiânia: AB Editora, 2000. p. 27-38.

Metodología de recolección de las representaciones sociales. In: ABRIC, JeanClaude (Org.). Prácticas sociales y representaciones. México: Ediciones Coyoacán, 2001. p. 53-74.

La recherche du noyau central et de la zone muette des représentations sociales. In: ABRIC, Jean-Claude. Méthodes d'étude des représentations sociale. Ramonville Sant-Agne, France: Érès, 2003. p. 58-80.

ALVARENGA, Luiza M. C. A. A prática médica no Programa de Saúde da Família e sua contribuição para mudança do modelo tecnoassistencial em saúde: limites e possibilidades. 2005. Dissertação (Mestrado em Saúde Pública) - São Paulo, Faculdade de Saúde Pública, USP, 2005.

ALVES, Vânia S. Um modelo de educação em saúde para o Programa Saúde da Família: pela integralidade da atenção e reorientação do modelo. Interface: Comunicação, Saúde, Educação, Botucatu, v. 9, n. 16, p. 39-52, set. 2004/ fev. 2005.

ALVES, Vânia S.; NUNES, Mônica O. Educação em saúde na atenção médica ao paciente hipertenso no Programa Saúde da Família. Interface: Comunicação, Saúde, Educação, Botucatu, São Paulo, v. 10, n. 19, p. 131-147, jan./jun. 2006.

AMANCIO, Antonio S. C.; CARVALHO, Graças S.; RODRIGUES, Vitor M. C. P. Valores na educação em saúde e a formação profissional. Trabalho, Educação e Saúde, Rio de Janeiro, v. 10, n. 3, p. 527-540, nov./dez. 2012.

ARAÚJO, Flávia M. Ações de educação em saúde no planejamento familiar nas unidades de saúde da família do município de Campina Grande-PB. 2004. Monografia (Curso de especialização em Saúde da Família para Profissionais do Programa Saúde da Família de Campina Grande-PB) - Campina Grande, Núcleo de Estudos em Saúde Coletiva, Universidade Estadual da Paraíba e Secretaria Municipal de Campina Grande, 2004.

CAMPOS, Pedro H. F. A abordagem estrutural e o estudo das relações entre práticas sociais e representações sociais. In: CAMPOS, Pedro H. F.; LOUREIRO, Marcos C. S. (Org.). Representações sociais e práticas educativas. Goiânia: Ed. da UCG, 2003. p. 21-36. 
GASTALDO, Denise. É a educação em saúde saudável?. Educação e Realidade, Porto Alegre, v. 22, n. 1, p. 47-168, jun./jul. 1997.

GAZZINELLI, Maria F.; PENNA, Cláudia. Conhecimentos, representações sociais e experiência da doença. In: GAZZINELLI, Maria F. et al. (Org.). Educação em saúde: teoria, método e imaginação. Belo Horizonte: Editora UFMG, 2006. p. 25-33.

L'ABBATE, Solange. Educação em saúde: uma nova abordagem. Cadernos de Saúde Pública, Rio de Janeiro, v. 10, n. 4, p. 481-90, out./dez., 1994.

MARQUES, Sérgio C. et al. AIDS e Representações sociais: uma análise comparativa entre subgrupos de trabalhadores. Psicologia: Teoria e Prática, São Paulo, v. esp, n. esp, p. 91-104, 2004.

MARSIGLIA, Regina M. G. Auxiliares de enfermagem: mercado de trabalho, satisfação e expectativas no Programa de Saúde da Família de São Paulo. Trabalho, Educação e Saúde, Rio de Janeiro, v. 4, n. 1, jan./abr. 2006.

MOSCOVICI, Serge. Representações sociais: investigações em psicologia social. 2. ed. Petrópolis: Vozes, 2004. 404 p.

MOURA, Escolástica R. F.; SOUSA, Rosiléa A. Educação em Saúde Reprodutiva: proposta ou realidade do Programa Saúde da Família?. Cadernos de Saúde Pública, Rio de Janeiro, v. 18, n. 6, p. 1809-1811, nov./dez. 2002.

NUNES, Mônica O. et al. O agente comunitário de saúde: construção da identidade desse personagem híbrido e polifônico. Cadernos de Saúde Pública, Rio de Janeiro, v. 18, n. 6, p. 1639-1646, dez. 2002.

OLIVEIRA, Denize C. A promoção da saúde da criança: análise das práticas cotidianas através do estudo de representações sociais. 1996. Tese (Doutorado em Saúde Pública) - São Paulo, Faculdade de Saúde Pública da Universidade de São Paulo, SP, 1996.
O conceito de necessidades humanas e de saúde e sua articulação no campo das representações sociais. In: OLIVEIRA, Denize C.; CAMPOS, Pedro H. F. (Org.). Representações sociais: uma teoria sem fronteiras. Rio de Janeiro: Editora Museu da República, 2005. p. 119-139.

OLIVEIRA, Denize C. et al. Análise das evocações livres: uma técnica de análise estrutural das representações sociais. In: MOREIRA, Antonia S. P.; CAMARGO, Brígido V.; JESUÍNO, Jorge C.; NÓBREGA, Sheva M. (Org.). Perspectivas teórico-metodológicas em representações sociais. João Pessoa: Editora Universitária UFPB, 2005. p. 573-603.

PINAFO, Elisangela; NUNES Elisabete F. P.; GONZÁLEZ, Alberto D.; GARANHANI, Mara L. Relações entre concepções e práticas de educação em saúde na visão de uma equipe de saúde da família. Trabalho, Educação e Saúde, Rio de Janeiro, v. 9, n. 2, p. 201-221, jul./out. 2011.

PEDROSA, José I. S.; TELES, João B. M. Consenso e diferenças em equipes do Programa Saúde da Família. Revista de Saúde Pública, São Paulo, v. 35, n. 3, p. 303-311, jun. 2001

SÁ, Celso P. Núcleo central das representações sociais. 2 ed. Petrópolis: Vozes, 2002.

SHIMIZU, Helena E. et al. A prática do auxiliar de enfermagem no programa saúde da família. Revista Latino-Americana Enfermagem, Ribeirão Preto, v. 12, n. 5, p. 713-720, set./out. 2004.

SILVA, Joana A.; DALMASIO, Ana S. W. Agente comunitário de saúde: o ser, o saber, o fazer. Rio de Janeiro: Editora Fiocruz, 2002.

SILVA, Maria A. et al. Enfermeiro \& grupos em PSF: possibilidade para participação social. Cogitare Enfermagem, Curitiba, v. 11, n. 2, p. 143-149, maio/ago. 2006. 
VERGÈS, Pierre. Ensemble de programmes permettant: version 2. Aix-en-Provence: Lames, 2000.

WAGNER, Wolfgang. Sócio-gênese e características das representações sociais. In: MOREIRA, Antônia S. P.; OLIVEIRA, Denise C. (Org.). Estudos interdisciplinares de representação social. 2 ed. Goiânia: AB Editora, 2000. p. 3-25.
WENDHAUSEN, Águeda; SAUPE, Rosita. Concepções de educação em saúde e a estratégia de saúde da Família. Texto \& Contexto Enfermagem, Florianópolis, v. 12, n. 1, p 17-25, jan./mar. 2003.

Recebido em 13/12/2011

Aprovado em 12/10/2012 